\title{
Music to my ears, goal for my eyes? Music reward modulates gaze disengagement from negative stimuli in dysphoria

\author{
Malvika Godara $^{1 *}$, Alvaro Sanchez-Lopez ${ }^{2}$, and Rudi de Raedt ${ }^{1}$ \\ ${ }^{1}$ Department of Experimental Clinical \& Health Psychology; Ghent University (Belgium) \\ ${ }^{2}$ Department of Clinical Psychology; Complutense University of Madrid (Spain)
}

*Corresponding Author:

Malvika Godara

Department of Experimental, Clinical \& Health Psychology

Ghent University

Henri Dunantlaan 2

Ghent 9000

Belgium

+3292649414

malvika.godara@ugent.be

Word Count (main text): 5,962 words

Keywords: Attention, negative information, eye-tracking, reward, goals, music

Declarations of interest: none

Data made available at osf.io/3xkab 


\begin{abstract}
Attentional bias for negative information, i.e. difficulties in disengagement from negative stimuli, is considered to be one of the core mechanisms involved in the onset and maintenance of depression. However, current attention training procedures aimed at reducing this bias have shown limited success. In addition to the reliability and validity concerns generated by the use of dot-probe paradigm in these procedures, an important factor is the limited consideration of motivational influences in the use of attention training. Therefore, in the current study we examined whether goal stimuli, reinforced with music reward, can modulate attention for negative information in dysphoric individuals. Using a novel attention task which measures gaze disengagement from negative faces, we found that dysphoric individuals displayed greater difficulties in disengaging eye-gaze from negative and directing it towards standard neutral stimuli, as compared to non-dysphorics. However, when using reward-reinforced goal stimuli, dysphoric individuals were as quick as non-dysphorics in disengaging attention from negative stimuli in order to engage with goal-related stimuli. These results provide preliminary evidence for the modulating role of music-reinforced goals in the attention system of depressed individuals, and highlight how music-reinforced goals can be incorporated in current attention training procedures to improve outcomes.
\end{abstract}




\section{Introduction}

Major Depressive Disorder (MDD) is a highly prevalent disorder, with nearly 322 million individuals suffering worldwide, and it is the leading cause of non-fatal health loss disability across the globe (WHO, 2017). It creates a significant economic and societal burden, with nearly 92 million euros spent each year in direct and indirect costs in the European Union alone (Olessen et al., 2012). Despite the urgency created by the adverse individual and societal costs, the current plethora of psychological interventions demonstrate only limited impact on depressive symptoms in the long run (Cuijpers et al., 2010). This underscores the need for a deeper understanding of the underlying mechanisms of depression, which can lead to the development of more well-informed interventions for the disorder.

One of the core mechanisms for depression is the presence of an attentional bias for negative information, more specifically the difficulty in disengaging attention from negative information in the environment (De Raedt \& Koster, 2010). Current models of depression (Disner, Beevers, Haigh \& Beck, 2011) posit a key role of attentional bias for negative information in the development and maintenance of the disorder. As negative mood states become activated by sustained attention to negative stimuli, it leads to activation of negative selfschemas (Farb, Irving, Anderson \& Segal, 2015; Watkins \& Nolen-Hoeksema, 2014). This in turn causes the maintenance of a chronic low mood, and enhanced attentional bias. In line with these models, elevated levels of attentional bias for negative information have been found to be associated with increased rumination and low reappraisal ability (Duque, Sanchez \& Vazquez, 2014; Everaert et al., 2017; Joormann \& Tanovic, 2015; Whitmer \& Gotlib, 2013), both hallmarks of depression. 
Eye-movement tracking procedures have been able to reliably delineate a stable attentional bias for negative information in depressed individuals (Armstorng \& Olantuji, 2012), including negative words (Koster, De Raedt, Leyman, \& De Lissnyder, 2010; Sanchez, Everaert, De Putter, Mueller, \& Koster, 2015) and negative emotional faces (Duque \& Vazquez, 2015; Sanchez et al., 2013). Sanchez and colleagues (2013) developed a novel eye-tracking task to measure processes of attentional (eye-gaze) engagement towards and disengagement from emotional faces in depressed individuals, assessing the impact of these processes on mood and recovery from stress. They presented 2 pictures to participants, one emotional (positive and negative) and one neutral of the same individual. In this task, after naturalistic viewing of the pair of faces, the task paused until the participants fixated their eye-gaze on one of the two images (predefined to be on the emotional or on the neutral one, depending on the trial). Upon fixation for $100 \mathrm{~ms}$ on that target image, a square or circle frame appeared around the other image. Participants had to direct their eye-gaze to the framed image, and respond, using one of two keys, whether they saw a square or a circle frame. This method allows to directly monitor and separately index processes of attentional engagement with emotional information (i.e., time to disengage gaze from neutral to make a new visual fixation on the opposite framed emotional image), and attentional disengagement from emotional information (i.e., time to disengage gaze from emotional to make a new visual fixation on the opposite framed neutral image). Sanchez and colleagues (2013) found that clinically depressed individuals, compared to healthy controls, had more difficulties in disengaging their attention from negative, but not positive, face expressions, specifically sad faces. Further, they found that this attentional bias for negative faces predicted poorer recovery in sad mood after undergoing a stress induction. Depressed 
individuals did not differ significantly from healthy controls in engagement towards either positive and negative emotional faces.

These difficulties in attentional disengagement from negative emotional faces are echoed by the results from studies conducted in dysphoric individuals (Koster, De Raedt, Goeleven, Franck, \& Crombez, 2005; Koster et al., 2010; Leyman, De Raedt, Vaeyens, \& Philippaerts, 2011; Sears, Thomas, LeHuquet, \& Johnson, 2010) and remitted depressed individuals (Albert, Gau, \& Taylor, 2017). Further, these effects have been replicated when also using the engagement-disengagement task to test time disengagement from negative faces and its relation with impaired stress recovery in dysphoric individuals (Sanchez, Romero \& De Raedt, 2017). This all-pervasive presence of an attentional bias for negative stimuli at every depressogenic state suggests that possible reductions in this bias can impact, and possibly diminish, depressive symptoms. However, current attention training procedures, termed Attention Bias Modification $(\mathrm{ABM})$, have demonstrated little to no success in reducing pre-existent depression-related attentional biases (Cristea, Kok \& Cuijpers, 2015; Mogoase, David \& Koster, 2014). ABM uses dot-probe based attention training, wherein participants see a pair of negative and neutral pictures. These pictures are replaced by a probe in the location of the neutral picture, and participants have to press a button to indicate the location as quickly as possible. Other than the fact that dot-probe based tasks and trainings have struggled with poor psychometrics (Zvielli et al., 2016), an important drawback of ABM may be the lack of a motivational component which could make the trainings more engaging (Beard, Sawyer \& Hoffman, 2012; Bjork, Dunlosky \& Kornell, 2013). This highlights a need for a deeper examination of the core mechanism of attentional bias for negative information, and an investigation into the possible methods through which this bias can be alleviated. 
All stimuli in the environment attract and compete for attention. However, depending upon the context, the most relevant stimuli are attended to and processed, creating a selective attention mechanism (Desimone \& Dunacan, 1995). This selective attention mechanism is guided by the goals of an individual within the context, such that goal-relevant stimuli attract more attention filtering out other goal-irrelevant stimuli (Allport, 1989; Corbetta \& Shulman, 2002; Vogt et al., 2011). Goals of individuals, in any given context, are dictated by their motivation to approach positive outcomes or avoid negative consequences (Carver \& Scheier, 1990; Trew, 2011). For example, reward-related stimuli exercise selective attention because the motivation to approach rewards transforms reward-related stimuli into salient approach goals (Chelazzi et al., 2013). Depressed individuals are characterized by imbalances between approach and avoidance motivation systems, tending to have more avoidance goals despite the context (Dickson \& MacLeod, 2006; Manos, Kanter \& Busch, 2010; Trew, 2011). Importantly, such motivational imbalances have been found to be associated to dysfunctional functioning in brain areas. Left frontal cortex hypoactivation is a hallmark in depression (Siegle, Thompson, Carter, Steinhauer, \& Thase, 2007) that precipitates approach deficits, leading to reduced positive information processing in depressed individuals (Trew, 2011). Further, amygdala abnormalities, decreased positive experiences and increased negative affect, in combination with rumination culminate in the presence of a negative information processing bias (Siegle, et al., 2007, Trew, 2011). As such, this suggests that an imbalance between approach and avoidance motivation might underlie a selective attention to and biased processing of negative information in depressed individuals. Therefore, increasing approach-oriented goals in depressed individuals might be key to altering aberrant attentional processes in depression. 
Recently, in the field of anxiety, several studies have used approach-oriented goals to override anxiety-related attentional biases for threatening stimuli. Vogt et al. (2013) compared attention to threat- and goal-related stimuli by presenting them together in a dot-probe task. They induced an approach goal through a goal inducer task, wherein participants had to press a button to earn points for a particular neutral image. The image became a goal-relevant image through its association with the reward. This goal-relevant image was then presented with threat-related and neutral images in the trials of the dot-probe task, which alternated with the trials of the goal inducer task. They found that even though an attentional bias for threat stimuli existed when threat-related and neutral pictures were presented together, this bias was overridden by selective attention for goal-related stimuli when threat- and goal-related stimuli were presented simultaneously. Similarly, a recent study (Sigurjónsdóttir et al., 2015), which incorporated rewards into $\mathrm{ABM}$ for social anxiety disorder, found evidence for influencing dysfunctional attention patterns for threat stimuli by using monetary rewards. Despite the difference between the attentional bias for threat in anxiety (engagement to threat) and the bias for negative information in depression (difficulty to disengage from negative stimuli), it might be of interest to examine whether approach-oriented goal stimuli create a similar attention prioritization in depressed individuals. Therefore, in the current study we examined whether approach-oriented goals, reinforced by reward, can create a stronger attentional bias for goal-related stimuli, in turn overriding the pre-existing attentional bias for negative stimuli in dysphoric individuals.

For the purpose of our study, we utilized the novel attention engagement-disengagement task developed by Sanchez and colleagues (2013). Using the disengagement condition of this task allowed us to test how swiftly participants were able to disengage their attention (gaze) from negative face expressions in order to engage attention towards either a standard neutral or 
reward-reinforced goal neutral stimuli. Akin to the design of Vogt et al. (2013), we alternated trials of the attention engagement-disengagement task with the trials of a goal inducer task, wherein a single neutral image was reinforced with reward upon a button-press. However, as depressed and dysphoric individuals seem to suffer from reduced sensitivity to monetary rewards (Barch, Pagliaccio \& Luking, 2015; Hervas \& Vazquez, 2013; Pizzagalli et al., 2008), reinforcing the goal stimulus with money or points would have likely not created an attentional bias for the goal stimulus. Depressed individuals tend to show reductions in monetary rewardrelated biases, and also display a reduced ability to learn from reward-guided trainings as the sensitivity to money or points is impaired (Kumar et al., 2008; Maddox et al., 2012; Pizzagalli et al., 2008). Moreover, in a study of our lab by Brailean et al. (2014), it was found that conditioning reward cues with points did not create an attentional bias for the reward cue in dysphoric individuals. Therefore, in our current study we used music as a reinforcer for the goal stimulus instead of secondary rewards.

Processing of music has been seemingly found to involve activation of sub-cortical brain regions such as Nucelus Accumbens, Ventral Tegmantal Area and Hypothalamus (Blood \& Zatorre, 2001; Menon \& Levitin, 2005), unlike monetary rewards which seem to deploy the dorsolateral and orbital frontal cortex (Knutson et al., 2003; Thut et al., 1997). However, unlike secondary rewards, music has not been used to initiate reward-based learning in depression yet. A recent study by Lazarov et al (2017), used music to successfully train socially anxious individuals to override their attentional bias for socially-threatening stimuli. Whereas music might be able to create a bias for rewarded cue in dysphoric individuals, it might not do so in non-dysphoric individuals. Previous research has demonstrated that healthy, non-dysphoric individuals seem to show a robust sensitivity to monetary rewards (Henriques \& Davidson, 2000; 
Pizzagalli et al., 2008; Pizzagalli, Jahn, \& O'Shea, 2005), which might make monetary reward more motivating for non-dysphoric individuals. For the purpose of our study, it was then necessary to induce a balanced incentive motivation in all participants (i.e., both dysphoric and non-dysphoric participants), such that approach motivation is engendered for the goal stimulus in both groups. Therefore, in the current study we decided to use both music and money as reinforcers for the goal stimulus.

In addition to investigating attentional disengagement from negative stimuli in 2 conditions, towards 'standard neutral' images and towards 'goal-related neutral' images, we added a third condition called 'action'. Since in our study the goal stimulus required a buttonpress to initiate the reward, it is possible that any attentional bias that we would find towards the goal stimulus might just be a function of the stimulus-response contingency created by the action of pressing the button (Bekkering \& Neggers, 2002). Therefore, in the goal inducer task we included another comparison condition, where participants were also required to press the button for another unique neutral image, but the button-press did not give them any reward. Therefore, when using this image from the 'action' condition in the attention engagement-disengagement task, we examined how quickly participants disengaged attention from the negative picture and then engaged towards the 'action-related neutral' image. This allowed us to determine whether the attentional bias for 'goal' images was greater than the bias created by the 'action' images.

In line with the extant literature on attentional bias for negative stimuli (De Raedt \& Koster, 2010), we expected that dysphoric individuals would have difficulties in disengaging attention from negative images and directing attention towards standard (i.e., no rewarded-goal) neutral images. Further, we investigated whether it is possible for goal-relevant neutral stimuli reinforced with music reward to override this attentional bias for negative stimuli in dysphoric 
individuals (i.e., faster times to disengage from negative when having to fixate on framed goalthan on framed standard-neutral faces). For non-dysphoric individuals, we did not expect any difficulties in disengaging attention from negative stimuli and directing it towards standard neutral or rewarded-goal stimuli.

\section{Method}

\section{Participants}

Fifty-nine individuals took part in the study (45 Females; $M_{\text {age }}=21.95, S D_{\text {age }}=3.57,18-$ 40 years). All participants were pre-selected from the Ghent University participant pool $(N=$ 134) based upon their scores on the Anhedonia Depression (AD) subscale of the Mood \& Anxiety Symptoms Questionnaire - Dutch (MASQ-D30; Wardenaar et al., 2010). A total of 59 individuals took part in the study, however, 1 participant was excluded from data analysis due to low quality of eye-gaze data obtained ( $<75 \%$ samples collected), and 1 participant was excluded due to detection of a psychiatric condition. Finally, 24 individuals formed the non-dysphoric group $\left(M_{M A S Q}=18.44, S D_{M A S Q}=2.97\right)$ and 33 individuals were included in the dysphoric group $\left(M_{M A S Q}=32.74, S D_{M A S Q}=5.94\right)$, as measured at the time of testing. Individuals were included in the dysphoric group if they scored in the top $25 \%$ of the participant pool $(>=26)$ on the AD scale of MASQ, and in the non-dysphoric group if they scored in the bottom 25\% (<=21). Participants were excluded if they had consumed drugs or alcohol within 6 hours prior to testing. Further, we excluded depressed patients because our aim was to investigate a proof-of-principle with minimum confounds such as medication use, prior episodes, cognitive problems. The study was approved by the ethical committee of the Faculty of Psychology \& Education Sciences at Ghent University. All participants provided informed consent and were compensated for their time $(€ 12)$. 


\section{Materials}

Questionnaires. Participants filled out the Dutch version of the 30-item short adaption of the Mood and Anxiety Symptoms Questionnaire (MASQ-D30; Wardenaar et al., 2010). This self-report measure is used to assess anxiety and depression symptoms on a 5 -point scale $(1=$ not at all, 5= extremely), out of which 10 items are depression-specific on the AD subscale. The items on the AD subscale are reverse scored. MASQ - D30 has a Cronbach's alpha of .92 in young adults, and in our sample the internal consistency was .93. Further, participants also underwent the MINI-International neuropsychiatric interview (Sheehan et al., 1998). The Mini is a short, structured diagnostic interview, which was used to confirm the presence or absence of any psychiatric conditions which the participants had not revealed to the experimenter, and which could otherwise interfere with our study. Finally, at the end of the experiment participants answered a single, self-report question: "Which reward, music or money, did you find more motivating?", and participants had to simply respond either "Music", "Money", or "Both".

Apparatus. The experiment, i.e. the stimulus presentation, was programmed using Eprime Professional 2.0 (2008). The stimuli were presented on a 23-inch high screen, which had a resolution of 1920 x 1080 pixels and a luminance of $300 \mathrm{~cd} / \mathrm{m}^{2}$. Participants were seated at a distance of $59-65 \mathrm{~cm}$ from the screen. We used the Tobii TX 300 eye tracker, which has a sampling rate of $300 \mathrm{~Hz}$ (binocular). E-prime stimulus presentations and eye-gaze recordings were merged into unified movie recordings using Tobii studio. These movie recordings contained the stimulus presentation as a movie with the pattern of eye movements made superimposed on top. The eye-gaze data from these recordings was converted into reaction times attention indices by marking out AOIs (Area of Interest) using Tobii Studio. 
Pictures. The stimuli (negative and neutral face expressions) were obtained from the Radboud Faces Database (RaFD; Langer et al., 2010). All pictures used in the current study consisted an individual, male or female, with a white background, facing towards the front. A total of 59 neutral pictures were taken from RaFD based on median scores on intensity $(M=3.4$, $S D=.3)$ and valence $(M=3.0, S D=.3)$, and constituted the 'standard neutral' condition. Two unique neutral picture from RaFD served as the 'goal' and the 'action' images $\left(M_{\text {intensity }}=3.3\right.$, $\left.S D_{\text {intensity }}=.3 ; M_{\text {valence }}=3.1, S D_{\text {valence }}=.3\right)$. The goal and action images were unique to their category and were not a part of the 'standard neutral' condition. Out of the 2 neutral images selected for those conditions, which picture served as the goal and action images was counterbalanced across participants. Sixty-one pictures constituted the 'negative' category (sad and disgust emotional faces) and were selected based on high scores for intensity ( $M=3.65 ; S D$ $=0.35)$ and valence $(M=2.05, S D=0.20)$. Pictures of all four categories, 'negative', 'standard neutral', 'goal' and 'action', were shown equally often throughout the entire experiment.

Music. During the goal inducer task, participants received reward in the form of music if they correctly identified the 'goal' image. The music used during this task consisted of one track that participants had to choose from a pre-selected list of 50 songs. The 50 songs consisted of tracks from 8 genres of music, and were selected based on the top charts in Belgium at the moment of running the experiment. Participants were told to select a song which made them happy and that they enjoyed listening to.

Goal Inducer Task. Each trial in the goal inducer task began with a black fixation cross $8 \mathrm{~mm}$ high, presented on a white background (see Figure 1). After 500ms, one image (157 x 237), from the 'negative', 'standard neutral', 'goal neutral' or 'action neutral' categories, appeared on the screen. If the image was from the 'negative' or 'standard neutral' categories, then the 
participants had to do nothing and the image would disappear after 1000ms. If the image belonged to the 'goal' or 'action' categories, then the participants had to press the spacebar as swiftly as possible. Upon button-press for the 'goal' image, participants received 062 cents and heard the music that they had picked for 10,000ms. If the participants did not press the spacebar for the 'goal' image within $1000 \mathrm{~ms}$ of the onset of the image or pressed the wrong button, they got the feedback 'Incorrect'. Upon button-press for 'action' image, participants did not receive any money or music, but if they forgot to press the spacebar or press the wrong button, the 'action' image stayed on the screen until the participants pressed the spacebar. A trial ended with a response (to the 'goal' or 'action' image) or when 1000ms had elapsed since the presentation of the picture on the screen. Participants performed 96 trials of the goal inducer task.

Attention engagement-disengagement task. Each trial in the attention engagementdisengagement task began with a black fixation cross ( $8 \mathrm{~mm}$ high) in the center of a white screen (see Figure 2). After participants fixated on the cross for 100ms, 2 pictures (157 x 237) appeared on the screen next to each other. The 2 pictures could be one of the following three combinations: 'negative'-'goal', 'negative'- 'action' or 'negative- standard neutral'. The 'negative', 'goal', 'action' and 'standard neutral' faces, and the male and female face expressions, were presented equally often on the left or right side of the screen. The 2 pictures were presented $10 \mathrm{~cm}$ apart from each other, and $5 \mathrm{~cm}$ each from the center of the screen. Participants were first instructed to freely attend to both the pictures presented on the screen, in the way they preferred. After 3,000ms freely viewing the faces pair, they completed the disengagement task: once the participants naturally made a new fixation on the negative picture for $100 \mathrm{~ms}$, a green square or circle frame appeared around the other (standard neutral, goal or action) picture. They had to direct their eye-gaze, as swiftly as possible, to the framed image and 
detect the frame surrounding the facial expression of that image. Participants pressed ' 1 ' if the frame was square or ' 2 ' if the frame was a circle. Both types of frames were presented equally often. Upon button-press, the trial elapsed. According to the three combinations of picture presented, the task comprised of three conditions: (1) 'Disengage to standard neutral': participants had to disengage attention from the negative picture and direct eye-gaze towards a standard (neither action nor goal-associated) neutral picture, (2) 'Disengage to action': participants had to disengage attention from the negative picture and direct eye-gaze towards the action-related neutral picture, and (3) 'Disengage to goal': participants had to disengage attention from the negative picture and direct eye-gaze towards the goal-related music-rewarded neutral picture. Participants performed a total of 16 trials each of the 'Disengage to goal' and 'Disengage to action' conditions, and 32 trials of the 'Disengage to standard neutral' condition. The unequal number of trials were presented so as to make sure that each picture category was presented equally often throughout the entire experiment. In the goal inducer task, the 'goal' and 'action' neutral images were presented twice as often as the 'negative' and 'standard neutral' images. Therefore, to balance the presentation of all pictures throughout the experiment, 16 additional trials of the 'Disengage to standard neutral' condition were presented during the attention engagement-disengagement task.

Further, in order to make sure that performance in the main disengagement trials was not compromised by participants being able to anticipate the location of the frame, they also performed 32 trials of the attention engagement condition (Sanchez et al., 2013). Trials of the attention engagement condition were similar to the attention disengagement condition, except participants had to, first, naturally fixate on the 'standard neutral', 'goal' or 'action' neutral image. After a fixation for $100 \mathrm{~ms}$ on such stimuli, the square or circle frame appeared around the 
opposite negative image. Participants had to disengage attention from the current image in order to engage attention toward the negative face, and press either ' 1 ' or ' 2 ' to indicate the type of frame. Each trial of the attention engagement-disengagement task was alternated with a trial of the goal inducer task (see Figure 3 for combined trial procedure).

\section{Procedure}

The experiment began with a brief explanation of the study, followed by participants filling out the informed consent and completing the questionnaires. Upon undergoing the calibration procedure for eye-tracking, participants were introduced to the attention engagementdisengagement task. Participants first performed 32 trials of the attention engagement condition to familiarize them with the protocol of the attention task, yet keeping them naïve about the exact procedure of the disengagement condition. Next, participants were introduced to the goal inducer task. They were instructed to press the spacebar for 2 specific pictures (goal- and action-related neutral images), and were informed that button-press for one image will reward them with their chosen music while the other one will not. After the instructions, participants performed 10 trials of the goal inducer task. Next, participants were informed that the trials of the two tasks would be presented together, one after the other. Before moving on to the main experimental trials, participants performed 20 trials of the combined attention disengagement and goal inducer tasks (see Figure 3 for trial procedure of the combined task). Then, all trials in the main combined task were presented in a pseudo-randomized order. Upon the completion of the main task, participants provided a self-report of measure of which reward, music or money, did they find to be more motivating. Finally, participants were debriefed and compensated for their time.

\section{Data Preparation}


The times to make a first visual fixation on the framed neutral image were obtained from the eye-gaze recordings for every disengagement trial in the attention engagementdisengagement task. Average time to make a first visual fixation on the framed neutral image were computed separately for each of the three conditions (negative to standard neutral, negative to action, negative to goal), to have an index of how quickly participants were able to disengage attention from negative picture and direct it towards standard neutral, action and goal neutral images. Before calculating the indices for the three conditions, we removed the outliers. All times to first fixate the framed image less than $100 \mathrm{~ms}$ or greater than $1000 \mathrm{~ms}$ were set to missing, as well as all scores with a value of 0 or '-' (missing values) (6\% removed in total). Upon performing data reduction, three first fixation indices were calculated by taking a mean of all the time to first fixation scores in each condition: "Disengage to standard neutral', 'Disengage to action', and 'Disengage to goal'. Disengagement indices demonstrated excellent psychometric properties. The internal consistency for trials for each of the three conditions was as follows: "Disengage to standard neutral" $(\alpha=.99)$, "Disengage to action" $(\alpha=.94)$, and "Disengage to goal" $(\alpha=.93)$.

\section{Results}

A 2 (Group) x 3 (Condition) mixed analysis of variance (ANOVA) was conducted using the three time to first fixation indices. Group (Dysphoric vs. Non-Dysphoric) was the betweensubjects variable, and Condition ('Disengage to standard neutral, 'Disengage to action' and 'Disengage to goal') was the within-subjects factor. We found a significant main effect of 
Condition, $F(2,110)=105.98, p<.001, \eta_{G}{ }^{2}=.42^{1}$, and a main effect of Group, $F(1,55)=75.71$, $p<.001, \eta_{G}{ }^{2}=.46$. More importantly, these effects were qualified by a significant interaction between Group and Condition, $F(2,110)=109.65, p<.001, \eta_{G}{ }^{2}=.43^{2}$.

Significant Bonferroni-corrected pairwise between-group comparisons confirmed that dysphoric individuals had significantly longer first fixation times on the framed image in the 'Disengage to standard neutral condition', in comparison to non-dysphorics $(p<.001)$. This is indicative of more difficulties to disengage gaze from negative stimuli in dysphorics (see Figure 4). Within-group comparison revealed that dysphoric individuals had significantly shorter time to first fixation to the framed image in 'Disengage to action' condition compared to the 'Disengage to standard neutral condition' $(p<.001)$. Further, between-group comparisons showed that dysphorics, compared to non-dysphorics, also had significantly longer times to first fixate the framed image in the 'Disengage to action' condition $(p<.001)$, such that dysphorics were slower in disengaging attention from negative stimuli to direct eye-gaze towards action stimulus, compared to non-dysphorics. However, in line with our hypothesis, pairwise comparisons showed that there were no significant differences between dysphoric and non-dysphoric individuals in the times to first fixate on the framed image in the 'Disengage to goal' condition, $p$ $=.66$. These results suggest that dysphorics found it as easy as non-dysphorics to disengage their attention from negative stimuli and direct it towards standard neutral stimuli, when the latter ones were goal-related stimuli. Confirming this effect, Bonferroni-corrected within-group comparisons revealed that, for dysphorics, the first fixation times on the framed image in the

\footnotetext{
${ }^{1}$ The generalized eta-squared has been calculated for both the main effects and the interaction effect. The generalized eta-squared allows for comparison with studies where similar between-subjects design might not be used, however still accounting for individual differences (Lakens, 2013).

2 The partial eta-squared for the effects are as follows: main effect of condition $\left(\eta_{p}^{2}=.66\right)$, main effect of group $\left(\eta_{p}^{2}\right.$ $=.58)$, and the interact effect condition $\mathrm{x}$ group $\left(\eta_{p}^{2}=.67\right)$.
} 
condition 'Disengage to Goal' were significantly faster than in both the 'Disengage to standard neutral', $p<.001$, and 'Disengage to action', $p<.001$ conditions. In contrast, pairwise comparisons for the non-dysphoric group revealed that there were no significant differences between the first fixation times on the framed image for the three conditions, all $p$ 's $>.10$.

Self-report data pertaining to the question "Which reward, music or money, did you find more motivating?" were aggregated. $81 \%$ of the participants ( 24 of 33 individuals) in the dysphoric group reported that they found music to be the most motivating, while, in contrast, nearly $89 \%$ of the participants ( 21 of 24 individuals) in the non-dysphoric group reported money to be a stronger motivator. Both money and music were reported to be found motivating by $12 \%$ of the dysphoric and $8 \%$ of the non-dysphoric individuals. These self-report results suggest that dysphoric individuals largely displayed a preference for music as a motivator, while, in line with previous research (Henriques \& Davidson, 2000; Pizzagalli et al., 2008; Pizzagalli, Jahn, \& O'Shea, 2005), non-dysphorics showed a stronger motivation towards monetary rewards.

\section{Discussion}

Considerable previous research has provided evidence for a pathogenic role of attentional bias for negative information in depression (De Raedt \& Koster, 2010). Specifically, difficulties to disengage attention from negative information have been found to predict impaired stress regulation in both clinically and subclinically depressed individuals (Sanchez et al., 2013, 2017). Yet, attention training procedures focused on reducing depression-related negative attentional biases have been largely unsuccessful (Cristea et al., 2015). The main aim of this study was to examine whether approach goal-related stimuli, compared to neutral stimuli, could help dysphoric individuals to disengage their attention from negative stimuli. In line with our predictions, we found that dysphoric individuals were faster to disengage their attention from 
negative faces when negative pictures were presented with goal-related pictures which had been reinforced with music, in comparison to regular non-rewarded neutral pictures.

Corroborating previous research, we found an attentional bias for negative stimuli in dysphoric individuals (De Raedt \& Koster, 2010; Sanchez et al., 2013, 2017). Dysphoric compared to non-dysphoric individuals were slower to disengage their attention from negative stimuli when they were presented with both standard, non-rewarded neutral stimuli, and actionrelated stimuli. Further, according to within-group comparisons, we found that dysphoric individuals were faster to disengage from negative stimuli and direct their attention towards action-related images than directing their attention to standard neutral stimuli. This suggests that performing an action for an image created an attentional bias for the stimulus (Bekkering \& Neggers, 2002), which then competed for attentional priority with the attentional bias for negative stimuli in dysphoric individuals. Meanwhile, given the absence of an attentional bias for negative stimuli in non-dysphoric individuals, there were no significant differences between disengaging attention towards standard neutral or action images. Finally, we also found that dysphoric individuals were faster to disengage attention from negative stimuli and direct it towards the goal stimulus, compared to standard neutral or action images. Importantly, this ease of disengagement was comparable in dysphoric and non-dysphoric individuals, such that there were no significant differences between groups in their attention disengagement from negative stimuli when prompted to engage with goal-related information.

These findings have implications for theories of attentional bias in the context of dysphoria and depression. The current view surrounds sustained attention to negative information, such that depressed individuals find it difficult to disengage attention from negative information in their environment (De Raedt \& Koster, 2010). The results from the current study 
suggest that attention for negative information can be modulated by approach-oriented goals. This implies that the attention processes in depression can be flexible depending upon the different goals activated in the situation. Based upon which goal holds priority, attentional system prioritizes the goal-relevant stimuli (Vogt et al., 2010).

These results also have implications for the current treatment of depressed individuals. Attentional bias for negative information has been espoused to be one of the causing and maintaining factors of depression, implying that reducing negative attentional bias may be a key target for therapeutic interventions. Standard attention training procedures, such as dot-probe based ABM, train depressed individuals to look away from negative stimuli by repeatedly directing their attention towards neutral images using the dot-probe paradigm. However, as discussed previously, these procedures have yielded limited success to modify attention (Cristea et al., 2015). Therefore, applying the results from the current study to attention training procedures can probably produce stronger effects. In future studies in depression, attention could be trained away from negative information by using approach goal-related stimuli which have been previously conditioned with reward, replacing simple neutral stimuli. For example, in a dotprobe based ABM training, depressed individuals could be trained to direct their attention away from negative stimuli by repeatedly directing it towards neutral stimuli which have been reinforced with rewarding music. Approach goals would likely exercise attention prioritization effects if conditioned with music reward, as suggested by the self-report results from the current study. This would most likely produce sustained changes in attention patterns, and could also impact other indicators or symptoms of depression as the motivational influence of goal cues is stronger than that of neutral stimuli. 
A few limitations of the present research must be considered, and need to be addressed by future studies. First, the current study was conducted in a sample of dysphoric individuals, who at the time of testing showed elevated depressive symptoms. As such, the results need to be replicated in a sample of clinically depressed individuals to make a conclusive claim on the modulating effect of approach goals on attention. Further, in our study we used both the application of music and money gain to reinforce the goal stimulus. This limits our ability to fully disentangle the rewarding influence of both music and money gain in the formation of approach goals. However, the results from the motivator self-report provide some clarity as to the impact of each type of motivator for the dysphoric and non-dysphoric individuals, results which are in line with previous research on monetary reward sensitivity in depressed and healthy individuals (Pizzagalli et al., 2008). Nevertheless, future studies are needed to clarify the differential impact of these motivators for both depressed and non-depressed populations. Finally, in the present study we did not consider how associated processes such as rumination tendencies or specific reward-sensitivity processes might account for individual differences in the observed attention disengagement patterns. For example, high ruminators are characterized by preferential sustained attentional processing of negative faces (see, for instance, Duque, Sanchez \& Vazquez, 2014), which might be accounted by more difficulties, compared to low ruminators, to disengage from negative faces and, consequently, to attend to goal stimuli, despite responding similarly to the motivators in the study. In other words, depression-related factors other than depression status itself might differentially influence the manifestation of negative disengagement impairments and responsiveness to reward manipulations. Therefore, future research is needed to examine how other depression-related factors impact the modulation of depression-related attention impairments by approach goals. 
Another important avenue for future research would be to test whether reward- and lossoriented goals modulate attention to emotional information in similar manner. In the current study, approach goals were induced by rewarding participants with music and money. However, goals associated with avoiding negative consequences can be important and motivating too. Therefore, it can be relevant to investigate the effect of avoidance goals on emotional attention as well. Further, it is possible that reward- and loss-sensitivity might play a key role in how approach and avoidance goals are formed and deployed. It is possible that individuals who value avoiding negative consequences more than gaining positive outcomes, might show a stronger impact of avoidance goals on emotional attention. Future studies should investigate this by incorporating a separate goal condition, wherein participants will have to avoid punishment (e.g., listening a white noise) or monetary loss.

In conclusion, the current study provides evidence for the idea that approach-oriented goals, conditioned with reward, can modulate the existing attentional bias for negative information in dysphoric individuals. These results provide a preliminary evidence for evaluative conditioned stimuli in supplanting the chronic attentional priority for negative information in depression. These findings have crucial implications for clinical interventions, and the application of these results might help to improve current attention trainings. 


\section{Acknowledgements}

This research was supported by Grant BOF16/GOA/017 for a Concerted Research Action of Ghent University awarded to Rudi De Raedt. Alvaro Sanchez-Lopez is supported by the Program for the Attraction of Scientific Talent of the Community of Madrid. The funding authorities were not involved in the conceptualization of this study, nor in the collection, analysis or writing up of the data. Further, the funding authorities were not involved in the decision to submit this manuscript for publication. 


\section{References}

Albert, K., Gau, V., Taylor, W. D., \& Newhouse, P. A. (2017). Attention bias in older women with remitted depression is associated with enhanced amygdala activity and functional connectivity. Journal of Affective Disorders, 210, 49-56. doi: 10.1016/j.jad.2016.12.010

Allport, A. (1989). Visual attention. In M. I. Posner (Ed.), Foundation of cognitive science (pp. 631-682). Cambridge, MA: MIT Press.

Armstrong, T., \& Olatunji, B. O. (2012). Eye tracking of attention in the affective disorders: A meta-analytic review and synthesis. Clinical Psychology Review, 32, 704-723. doi: 10.1016/j.cpr.2012.09.004.

Barch, D. M., Pagliaccio, D., \& Luking, K. (2015). Mechanisms underlying motivational deficits in psychopathology: similarities and differences in depression and schizophrenia. In Behavioral neuroscience of motivation (pp. 411-449). Springer, Cham. doi:

10.1007/7854_2015_376

Beard, C., Sawyer, A. T., \& Hofmann, S. G. (2012). Efficacy of attention bias modification using threat and appetitive stimuli: A meta-analytic review. Behavior Therapy, 43, 724-740. doi: 10.1016/j.beth.2012.01.002

Bekkering, H., \& Neggers, S. F. (2002). Visual search is modulated by action intentions. Psychological Science, 13, 370-374. doi:10.1111/j.0956-7976.2002.00466.x

Bjork, R. A., Dunlosky, J., \& Kornell, N. (2013). Self-regulated learning: Beliefs, techniques, and illusions. Annual Review of Psychology, 64, 417-444. doi: 10.1146/annurevpsych-113011-143823. 
Blood, A. J., \& Zatorre, R. J. (2001). Intensely pleasurable responses to music correlate with activity in brain regions implicated in reward and emotion. Proceedings of the National Academy of Sciences, 98, 11818-11823. doi: 10.1073/pnas.191355898

Brailean, A. M., Koster, E. H., Hoorelbeke, K., \& De Raedt, R. (2014). Attentional modulation by reward and punishment cues in relation to depressive symptoms. Journal of Behavior Therapy and Experimental Psychiatry, 45, 351-359. doi: 10.1016/j.jbtep.2014.03.003

Carver, C. S., \& Scheier, M. F. (1990). Origins and functions of positive and negative affect: A control-process view. Psychological Review, 97, 19.

Chelazzi, L., Perlato, A., Santandrea, E., \& Della Libera, C. (2013). Rewards teach visual selective attention. Vision Research, 85, 58-72. Doi: 10.1016/j.visres.2012.12.005.

Corbetta, M., \& Shulman, G. L. (2002). Control of goal-directed and stimulus-driven attention in the brain. Nature Reviews Neuroscience, 3, 201-215. doi:10.1038/nrn755

Cristea, I. A., Kok, R. N., \& Cuijpers, P. (2015). Efficacy of cognitive bias modification interventions in anxiety and depression: meta-analysis. The British Journal of Psychiatry, 206, 716. Doi: 10.1192/bjp.bp.114.146761

Cuijpers, P., van Straten, A., Bohlmeijer, E., Hollon, S. D., \& Andersson, G. (2010). The effects of psychotherapy for adult depression are overestimated: a meta-analysis of study quality and effect size. Psychological Medicine, 40, 211-223. doi: 10.1017/S0033291709006114.

De Raedt, R., \& Koster, E. H. (2010). Understanding vulnerability for depression from a cognitive neuroscience perspective: A reappraisal of attentional factors and a new conceptual framework. Cognitive, Affective, \& Behavioral Neuroscience, 10, 50-70. doi: 10.3758/CABN.10.1.50. 
Desimone, R., \& Duncan, J. (1995). Neural mechanisms of selective visual attention. Annual Review of Neuroscience, 18, 193-222. doi:10.1146/annurev.ne.18.030195.001205

Dickson, J. M., \& MacLeod, A. K. (2006). Dysphoric adolescents' causal explanations and expectancies for approach and avoidance goals. Journal of Adolescence, 29, 177-191. doi: 10.1016/j.adolescence.2005.03.007

Disner, S. G., Beevers, C. G., Haigh, E. A., \& Beck, A. T. (2011). Neural mechanisms of the cognitive model of depression. Nature Reviews Neuroscience, 12, 467. doi: 10.1038/nrn3027.

Duque, A., Sanchez, A., \& Vazquez, C. (2014). Gaze-fixation and pupil dilation in the processing of emotional faces: The role of rumination. Cognition and Emotion, 28, 1347-1366. Doi: 10.1080/02699931.2014.881327.

Duque, A., \& Vázquez, C. (2015). Double attention bias for positive and negative emotional faces in clinical depression: Evidence from an eye-tracking study. Journal of Behavior Therapy and Experimental Psychiatry, 46, 107-114. doi: 10.1016/j.jbtep.2014.09.005.

Everaert, J., Grahek, I., Duyck, W., Buelens, J., Van den Bergh, N., \& Koster, E. H. (2017). Mapping the interplay among cognitive biases, emotion regulation, and depressive symptoms. Cognition and Emotion, 31, 726-735. doi: 10.1080/02699931.2016.1144561.

Farb, N. A., Irving, J. A., Anderson, A. K., \& Segal, Z. V. (2015). A two-factor model of relapse/recurrence vulnerability in unipolar depression. Journal of Abnormal Psychology, 124, 38. doi: 10.1037/abn0000031.

Henriques, J. B., \& Davidson, R. J. (2000). Decreased responsiveness to reward in depression. Cognition and Emotion, 14, 711-724. doi: 10.1080/02699930050117684 
Hervas, G., \& Vazquez, C. (2013). Low spirits keep rewards subdued: Decreases in sensitivity to reward and vulnerability to dysphoria. Behavior Therapy, 44, 62-74. doi: 10.1016/j.beth.2012.07.003

Joormann, J., \& Tanovic, E. (2015). Cognitive vulnerability to depression: examining cognitive control and emotion regulation. Current Opinion in Psychology, 4, 86-92. doi: 10.1016/j.copsyc.2014.12.006.

Knutson, B., Fong, G. W., Bennett, S. M., Adams, C. M., \& Hommer, D. (2003). A region of mesial prefrontal cortex tracks monetarily rewarding outcomes: characterization with rapid event-related fMRI. Neuroimage, 18, 263-272. Doi: 10.1016/S1053-8119(02)00057-5

Koster, E. H., De Raedt, R., Goeleven, E., Franck, E., \& Crombez, G. (2005). Moodcongruent attentional bias in dysphoria: maintained attention to and impaired disengagement from negative information. Emotion, 5, 446. doi: 10.1037/1528-3542.5.4.446

Koster, E. H., De Raedt, R., Leyman, L., \& De Lissnyder, E. (2010). Mood-congruent attention and memory bias in dysphoria: exploring the coherence among information-processing biases. Behaviour Research and Therapy, 48, 219-225. doi: 10.1016/j.brat.2009.11.004.

Kumar, P., Waiter, G., Ahearn, T., Milders, M., Reid, I., \& Steele, J. D. (2008). Abnormal temporal difference reward-learning signals in major depression. Brain, 131, 20842093. doi: 10.1093/brain/awn136

Lakens, D. (2013). Calculating and reporting effect sizes to facilitate cumulative science: a practical primer for t-tests and ANOVAs. Frontiers in Psychology, 4, 863. doi: 10.3389/fpsyg.2013.00863 
Langner, O., Dotsch, R., Bijlstra, G., Wigboldus, D. H., Hawk, S. T., \& Van Knippenberg, A. D. (2010). Presentation and validation of the Radboud Faces Database. Cognition and Emotion, 24(8), 1377-1388. doi: 10.1080/02699930903485076

Lazarov, A., Pine, D. S., \& Bar-Haim, Y. (2017). Gaze-contingent music reward therapy for social anxiety disorder: A randomized controlled trial. American Journal of Psychiatry, 174, 649-656. doi: 10.1176/appi.ajp.2016.16080894

Leyman, L., De Raedt, R., Vaeyens, R., \& Philippaerts, R. M. (2011). Attention for emotional facial expressions in dysphoria: An eye-movement registration study. Cognition and Emotion, 25, 111-120. doi: 10.1080/02699931003593827

Maddox, W. T., Gorlick, M. A., Worthy, D. A., \& Beevers, C. G. (2012). Depressive symptoms enhance loss-minimization, but attenuate gain-maximization in history-dependent decision-making. Cognition, 125(1), 118-124. doi: 10.1016/j.cognition.2012.06.011

Manos, R. C., Kanter, J. W., \& Busch, A. M. (2010). A critical review of assessment strategies to measure the behavioral activation model of depression. Clinical Psychology Review, 30, 547-561. doi: 10.1016/j.cpr.2010.03.008

Menon, V., \& Levitin, D. J. (2005). The rewards of music listening: response and physiological connectivity of the mesolimbic system. Neuroimage, 28, 175-184. doi: 10.1016/j.neuroimage.2005.05.053

Mogoaşe, C., David, D., \& Koster, E. H. (2014). Clinical efficacy of attentional bias modification procedures: An updated meta-analysis. Journal of Clinical Psychology, 70, 11331157. doi: $10.1002 /$ jclp.22081. 
Olesen, J., Gustavsson, A., Svensson, M., Wittchen, H. U., \& Jönsson, B. (2012). The economic cost of brain disorders in Europe. European Journal of Neurology, 19, 155-162. doi: 10.1111/j.1468-1331.2011.03590.x

Pizzagalli, D. A., Iosifescu, D., Hallett, L. A., Ratner, K. G., \& Fava, M. (2008). Reduced hedonic capacity in major depressive disorder: evidence from a probabilistic reward task.

Journal of Psychiatric Research, 43, 76-87. Doi: 10.1016/j.jpsychires.2008.03.001

Pizzagalli, D. A., Jahn, A. L., \& O'Shea, J. P. (2005). Toward an objective characterization of an anhedonic phenotype: A signal-detection approach. Biological Psychiatry, 57, 319-327. doi: 10.1016/j.biopsych.2004.11.026

Psychology Software Tools, Inc. [E-Prime 2.0.8.22]. (2008). Retrieved from http://www.pstnet.com

Sanchez, A., Everaert, J., De Putter, L. M., Mueller, S. C., \& Koster, E. H. (2015). Life is... great! Emotional attention during instructed and uninstructed ambiguity resolution in relation to depressive symptoms. Biological Psychology, 109, 67-72. doi:

10.1016/j.biopsycho.2015.04.007

Sanchez, A., Romero, N., \& De Raedt, R. (2017). Depression-related difficulties disengaging from negative faces are associated with sustained attention to negative feedback during social evaluation and predict stress recovery. PloS one, 12, e0175040. doi:

10.1371/journal.pone.0175040

Sanchez, A., Vazquez, C., Marker, C., LeMoult, J., \& Joormann, J. (2013). Attentional disengagement predicts stress recovery in depression: An eye-tracking study. Journal of Abnormal Psychology, 122, 303. doi: 10.1037/a0031529. 
Sears, C. R., Thomas, C. L., LeHuquet, J. M., \& Johnson, J. C. (2010). Attentional biases in dysphoria: An eye-tracking study of the allocation and disengagement of attention. Cognition and Emotion, 24, 1349-1368. doi: 10.1080/02699930903399319

Sheehan, D. V., Lecrubier, Y., Sheehan, K. H., Amorim, P., Janvas, J., Weiller, E., ..., \& Dunbar, G. C. (1998). The Mini-International Neuropsychiatric Interview (MINI): the development and validation of a structured diagnostic psychiatric interview for DSM-IV and ICD-10. Journal of Clinical Psychiatry, 59, 22-33.

Siegle, G. J., Thompson, W., Carter, C. S., Steinhauer, S. R., \& Thase, M. E. (2007). Increased amygdala and decreased dorsolateral prefrontal BOLD responses in unipolar depression: related and independent features. Biological psychiatry, 61, 198-209. doi: 10.1016/j.biopsych.2006.05.048

Sigurjónsdóttir, O., Björnsson, A. S., Ludvigsdóttir, S. J., \& Kristjánsson, A. (2015). Money talks in attention bias modification: Reward in a dot-probe task affects attentional biases. Visual Cognition, 23, 118-132. doi:10.1080/13506285.2014.984797

Thut, G., Schultz, W., Roelcke, U., Nienhusmeier, M., Missimer, J., Maguire, R. P., \& Leenders, K. L. (1997). Activation of the human brain by monetary reward. Neuroreport, 8 , $1225-1228$.

Trew, J. L. (2011). Exploring the roles of approach and avoidance in depression: An integrative model. Clinical Psychology Review, 31, 1156-1168. doi: 10.1016/j.cpr.2011.07.007

Vogt, J., De Houwer, J., Crombez, G., \& Van Damme, S. (2013). Competing for attentional priority: Temporary goals versus threats. Emotion, 13, 587-598. doi:10.1037/a0027204 
Vogt, J., De Houwer, J., \& Moors, A. (2011). Unintended allocation of spatial attention to goal- relevant but not to goal-related events. Social Psychology, 42, 48-55. doi:10.1027/18649335/a000042

Wardenaar, K. J., van Veen, T., Giltay, E. J., de Beurs, E., Penninx, B. W., \& Zitman, F. G. (2010). Development and validation of a 30-item short adaptation of the Mood and Anxiety Symptoms Questionnaire (MASQ). Psychiatry research, 179(1), 101-106. doi:

10.1016/j.psychres.2009.03.005

Watkins, E. R., \& Nolen-Hoeksema, S. (2014). A habit-goal framework of depressive rumination. Journal of Abnormal Psychology, 123, 24. doi: 10.1037/a0035540.

Whitmer, A. J., \& Gotlib, I. H. (2013). An attentional scope model of rumination. Psychological Bulletin, 139, 1036. doi: 10.1037/a0030923.

WHO (2017). Depression and other common mental disorders: Global health estimates. Geneva, Switzerland: World Health Organization.

Zvielli, A., Vrijsen, J. N., Koster, E. H. W., \& Bernstein, A. (2016). Attentional bias temporal dynamics in remitted depression. Journal of Abnormal Psychology, 125(6), 768-776. doi: $10.1037 / \mathrm{abn} 0000190$ 


\section{Figure Captions}

1. An example of a trial in the goal inducer task.

2. An example of a disengagement trial in the attention engagement-disengagement task.

3. The combined trial procedure for the engagement-disengagement and goal inducer tasks.

4. First fixation to the framed image scores for both dysphoric and non-dysphoric individuals for the three disengagement conditions. To Standard Neutral $=$ Disengage from negative to standard neutral image, To Action = Disengage from negative to action image , To Goal $=$ Disengage from negative to goal image, High $=$ Dysphorics, Low $=$ Non-Dysphorics. First fixation times presented in seconds (along the y-axis). ${ }^{*}=$ significant difference $(p<.001)$. 
Figure 1

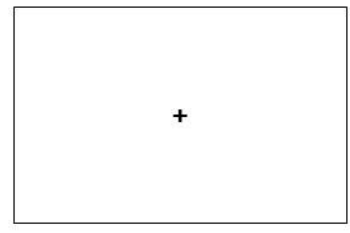

Fixation Cross (500ms)

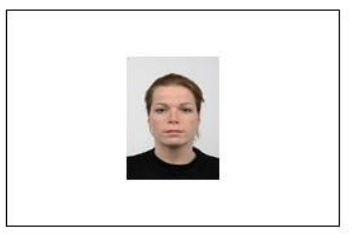

Presentation of Goal (2000ms), Non-Goal (2000ms) or Action (until response) stimuli

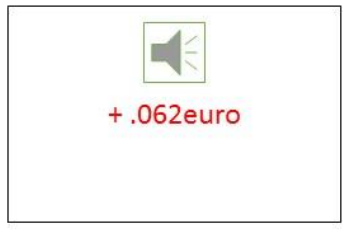

Reward for correct identification of Goal stimulus (10000ms) 
Figure 2

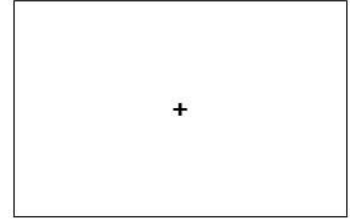

Fixation Cross (until fixation for $100 \mathrm{~ms}$ )

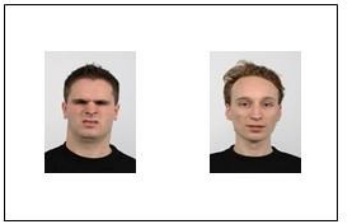

Naturalistic viewing of faces $(3000 \mathrm{~ms})$

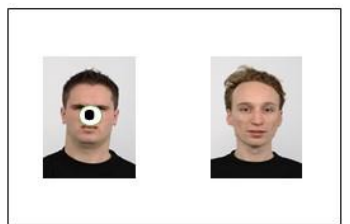

Wait for fixation on negative face $(100 \mathrm{~ms})$

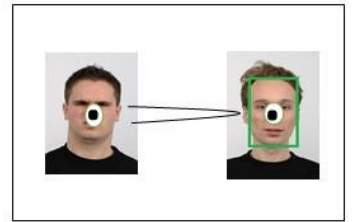

Response to the type of frame (until participant's response) 
Figure 3

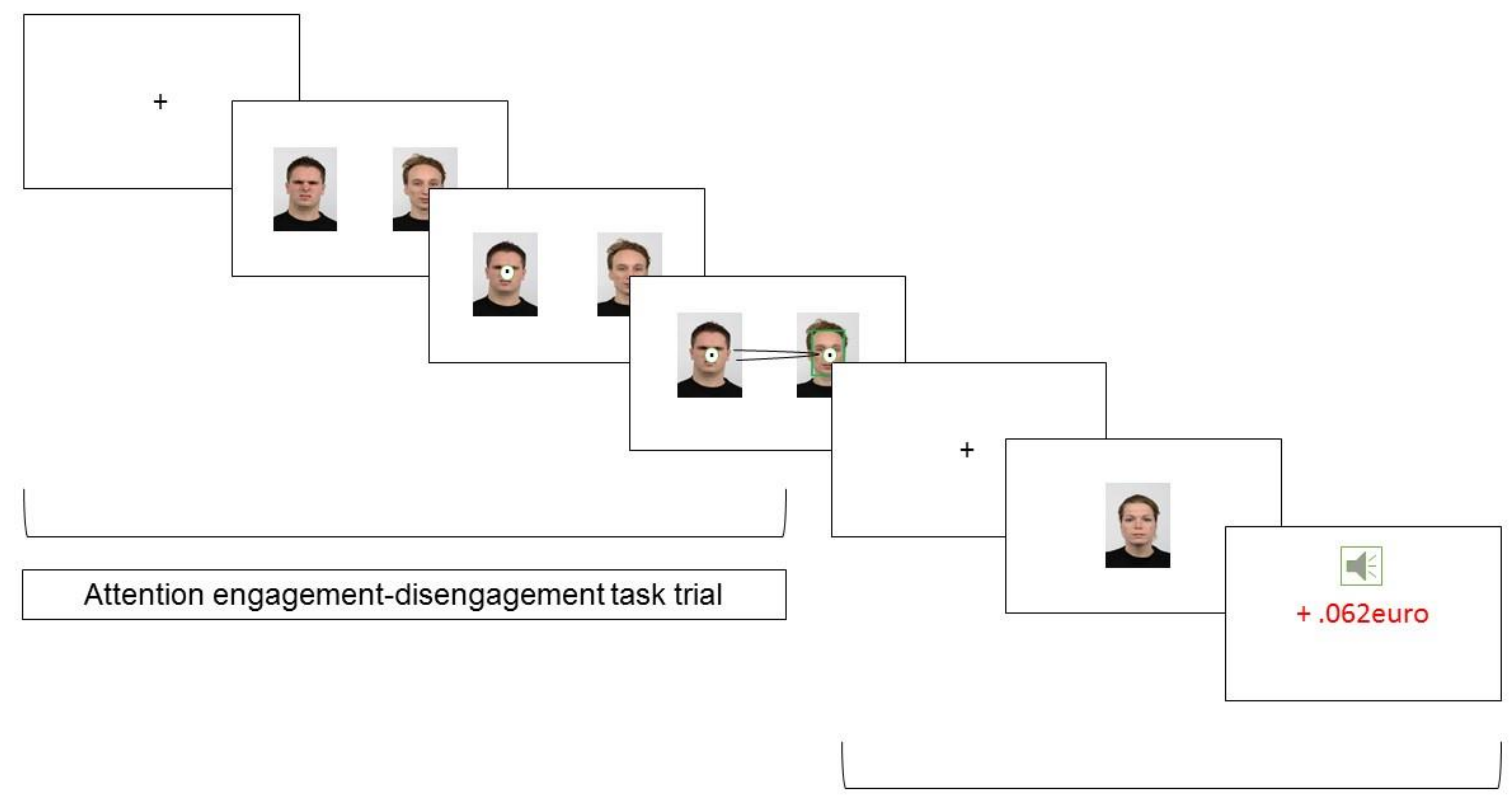

Goal inducer task trial 
Figure 4

\section{Disengaement from negative}

0,6

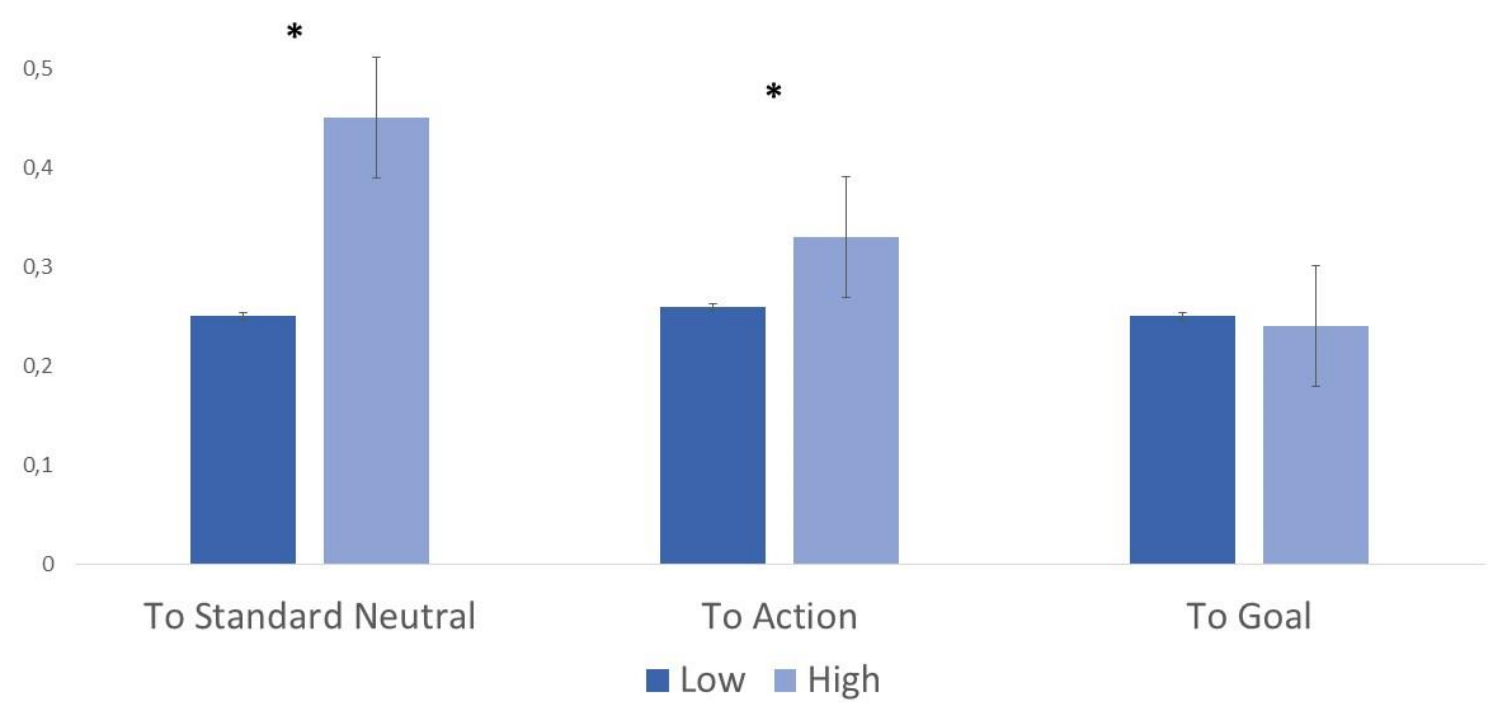

